\title{
Ab initio calculations of partial molar properties in the single-site approximation
}

\author{
Ruban, Andrei; Skriver, Hans Lomholt
}

Published in:

Physical Review B

Link to article, DOI:

10.1103/PhysRevB.55.8801

Publication date:

1997

Document Version

Publisher's PDF, also known as Version of record

Link back to DTU Orbit

Citation (APA):

Ruban, A., \& Skriver, H. L. (1997). Ab initio calculations of partial molar properties in the single-site approximation. Physical Review B, 55(14), 8801-8807. https://doi.org/10.1103/PhysRevB.55.8801

\section{General rights}

Copyright and moral rights for the publications made accessible in the public portal are retained by the authors and/or other copyright owners and it is a condition of accessing publications that users recognise and abide by the legal requirements associated with these rights.

- Users may download and print one copy of any publication from the public portal for the purpose of private study or research.

- You may not further distribute the material or use it for any profit-making activity or commercial gain

- You may freely distribute the URL identifying the publication in the public portal

If you believe that this document breaches copyright please contact us providing details, and we will remove access to the work immediately and investigate your claim. 


\title{
$A b$ initio calculations of partial molar properties in the single-site approximation
}

\author{
A. V. Ruban and H. L. Skriver \\ Center for Atomic-scale Materials Physics and Department of Physics, Technical University of Denmark, DK-2800 Lyngby, Denmark
}

(Received 23 October 1996)

\begin{abstract}
We discuss the application of the single-site approximation in calculations of partial molar quantities, e.g., impurity solution energy, segregation energy, and effective chemical potential, which are related to a variation of the composition of an alloy or its nonequivalent parts. We demonstrate that these quantities may be considerably in error if they are obtained in methods based on the single-site approximation for fixed alloy compositions. This error does not reflect a breakdown but rather an inappropriate use of the single-site approximation which is, in fact, found to be sufficiently accurate when properly applied in calculations of partial molar quantities. [S0163-1829(97)03713-2]
\end{abstract}

\section{INTRODUCTION}

The single-site approximation constitutes the basis of the key approximation in alloy theory: the coherent potential approximation (CPA). Recently, it has been shown that the CPA may give an accurate estimate of the energetics of random alloys provided one includes an approximate description of the charge distribution around an impurity which is undetermined in the single-site approximation. ${ }^{1}$ In spite of this success, there is still some doubt ${ }^{2,3}$ as to the validity of the single-site approximation itself. In particular, it has been shown by Drittler et al. ${ }^{2}$ that the impurity solution energies calculated by means of the Green's function technique in the single-site approximation may differ substantially from the values obtained when the perturbation of the neighboring sites is included. On the basis of these results it has been concluded that the single-site approximation is not reliable in general and may lead to considerable errors. ${ }^{2}$ Since this conclusion contradicts the results of Ref. 1, it is of great importance to find the reason for the apparent disagreement and perhaps reconcile the two approaches.

There is a second disagreement which at first sight appears unrelated to the impurity problem: The (100) surface concentration profiles of a random $\mathrm{CuNi}$ alloy obtained in two recent first-principles calculations differ not only quantitatively but also qualitatively. Ruban et $a l .{ }^{4}$ find an oscillatory concentration profile while Pasturel et $a .^{5}$ find a monotonic profile. The latter type of calculation has been repeated in a so-called self-consistent determination of the concentration profile by Drchal et al., ${ }^{6,7}$ again resulting in monotonic profiles. The disagreement between the two types of calculations appears very surprising in view of the fact that the same linear muffin-tin orbital ${ }^{8,9}$ (LMTO) and CPA methods are used, the only difference being that Pasturel et al. and Drchal et al. use the generalized perturbation method (GPM) to calculate concentration-dependent effective interactions, while Ruban et al. use the Connolly-Williams method (CWM) generalized to the case of a surface to calculate concentration-independent interactions. We note that the source of the disagreement is not to be found in the type of interactions used, i.e., concentration dependent or concentration independent, since it has been shown that they are in fact directly related. ${ }^{10,11}$ Hence, it must be found in the de- tails of the Connolly-Williams or the generalized perturbation methods.

In the present paper we show that the controversies have a common origin in the way the single-site approximation is applied in the calculation of partial molar properties such as the effective chemical potential, the impurity solution energy, and the segregation energy. In the GPM and similar methods based on fixed compositions the single-site approximation leads to a complete neglect of the relaxation of the electronic structure in the neighborhood of the perturbation. As already noted by Drittler et $a .^{2}$ such a neglect may be a serious approximation, and we will show that this is in fact the reason for the controversies. Further, we present one solution to the problem of calculating partial molar properties which involves the application of the CPA in conjunction with the CWM, as in the work by Ruban et al., ${ }^{4}$ whereby the electronic structure relaxation is naturally included and the accuracy of the calculations greatly improved. ${ }^{12-14}$

The paper is organized as follows. In Sec. II we outline and discuss in the most basic terms the theory which may be used to calculate partial molar properties from first principles. In particular, we emphasize the role of the single-site approximation. Part of this discussion may appear completely trivial. However, the points we raise have been overlooked in the literature for a long time, the most prominent example being the above-mentioned first-principles calculations of surface segregation profiles based on effective interactions obtained within the GPM (Refs. 5-7 and 15-17) or in the tight-binding direct configurational averaging (DCA) method. ${ }^{18}$ The task before us is therefore partly pedagogical. After a discussion of the connection between the two applications of the single-site approximation in calculations of partial molar properties we finish by substantiating our viewpoint by a numerical investigation of the random $\mathrm{CuNi}$ alloy system which is at the heart of the controversy.

\section{CALCULATIONS OF PARTIAL MOLAR PROPERTIES}

\section{A. Total energy of a random alloy}

Let us consider the simple example of a binary, completely random alloy $A_{1-c} B_{c}$ on a fixed lattice with equiva- 
lent sites and assume that the total energy per atom may be written in the form resembling the corresponding expression in the CPA:

$$
E_{\text {rand }}(c)=c E_{B}(c)+(1-c) E_{A}(c) .
$$

Here $c$ is the concentration of $B$, and the on-site energies $E_{B}(c)$ and $E_{A}(c)$ are the partial contributions to the total energy from the alloy components. We refer to this as the intensive, i.e., per atom, form for the total energy of a random alloy.

A decomposition of the total energy of a random alloy into partial contributions such as Eq. (1) is not possible in general. However, it exists, for instance, in the atomic sphere approximation $^{9}$ (ASA) in which the total energy of an alloy with an arbitrary configuration may be written in the extensive, i.e., per $N$ atoms, form

$$
\widetilde{E}_{\mathrm{tot}}=\sum_{i=1}^{N} \epsilon_{i},
$$

where $N$ is the number of atoms in the system and $\epsilon_{i}$ is the local contribution to the total energy from an atom at site $i$. Hence, we may use the ASA or a similar approximation, which like the ASA is sufficiently accurate in calculations of total energies as long as the geometry of the underlying lattice is fixed. In this case the on-site energies are determined as the average quantities

$$
\begin{gathered}
E_{B}(c)=\frac{1}{N_{B}} \sum_{i}\left\langle\epsilon_{i}(c) c_{i}\right\rangle=\left\langle\epsilon_{i}^{(i=B)}(c)\right\rangle, \\
E_{A}(c)=\frac{1}{N_{A}} \sum_{i}\left\langle\epsilon_{i}(c)\left(1-c_{i}\right)\right\rangle=\left\langle\epsilon_{i}^{(i=A)}(c)\right\rangle,
\end{gathered}
$$

and the total energy of a random alloy Eq. (1), may be written as

$$
E_{\text {rand }}(c)=\frac{1}{N} \sum_{i}\left\langle\epsilon_{i}(c)\right\rangle=\frac{1}{N}\left\langle\widetilde{E}_{\mathrm{tot}}(c)\right\rangle .
$$

Here $N_{A}$ and $N_{B}$ are the number of $A$ and $B$ atoms in the system, $N=N_{A}+N_{B}, c=N_{B} / N, c_{i}$ is an occupation number taking on the values 1 and 0 depending on whether site $i$ is occupied by a $B$ atom or an $A$ atom, respectively, and \langle\rangle designates configurational averaging over ensemble.

\section{B. Impurity solution energy}

We may now determine the solution energy of a $B$ atom in a pure $A$ crystal. This may be obtained in two ways, the first of which is based on the intensive form (1) of the total energy of a completely random $A_{1-c} B_{c}$ alloy. From the definition of a partial molar property we find

$$
E_{\mathrm{sol}}^{\mathrm{int}}(c) \equiv \frac{d E_{\mathrm{mix}}}{d c}=\frac{d E_{\mathrm{rand}}}{d c}-E_{B}(1)+E_{A}(0),
$$

where

$$
E_{\text {mix }}=E_{\text {rand }}(c)-\left[c E_{B}(1)+(1-c) E_{A}(0)\right]
$$

is the mixing energy of the alloy, and $E_{B}(1)=\epsilon_{i}(1)$ and $E_{A}(0)=\epsilon_{i}(0)$ are the total energies per atom of the pure alloy components. In the dilute limit of a single $B$ impurity in pure a $A$ host we find

$$
E_{\mathrm{sol}}^{\mathrm{int}}(0)=E_{B}(0)-E_{B}(1)+\left.\frac{d E_{A}(c)}{d c}\right|_{c=0},
$$

which we will refer to as the intensive definition of the impurity solution energy.

The second expression for the solution energy is based on the extensive form (1) of the total energy of a single crystal with $N$ atoms. In this picture the impurity solution energy, which is the energy it costs to move a $B$ atom from a pure $B$ crystal into site $i$ of a pure $A$ crystal, may be obtained as the difference in total energy of an $A$ crystal with a single $B$ impurity and that of a the pure $A$ crystal from which one subtracts the energy per atom, $E_{B}(1)=\epsilon_{i}(1)$, of a $B$ atom in a pure $B$ crystal. We have

$$
\begin{aligned}
E_{\mathrm{sol}}^{\mathrm{ext}}(0) & =\widetilde{E}_{\mathrm{tot}}(A+B)-\widetilde{E}_{\mathrm{tot}}(A)-E_{B}(1) \\
& =E_{B}(0)-E_{B}(1)+\sum_{j \neq i}\left[\epsilon_{j}^{(i=B)}-E_{A}(0)\right],
\end{aligned}
$$

where we have used the fact that in the thermodynamic limit $N \rightarrow \infty$ the energy, of a $B$ atom in a pure $A$ crystal, $\epsilon_{i}^{(i=B)}=E_{B}(0)$. Note that the summation over the remaining sites in the second line of Eq. (8) includes the important relaxation of the electronic structure of the neighboring $A$ atoms due to their interaction with the $B$ atom at site $i$. The extensive definition (8) is exactly the definition used in the successful Green's function calculations by Drittler et $a l^{2}$ of the impurity solution energy of the $3 d$ metals in $\mathrm{Cu}$ and $\mathrm{Ni}$.

If we compare Eqs. (7) and (8), we find the important relation

$$
\left.\frac{d E_{A}(c)}{d c}\right|_{c=0}=\sum_{j \neq i}\left[\epsilon_{j}^{(i=B)}-E_{A}(0)\right],
$$

connecting intensive and extensive quantities. The physical interpretation of Eq. (9) is transparent: The change of the on-site energies with concentration is equal to the renormalization of the on-site energies in the whole system with a single impurity.

We now introduce the single-site approximation which is the assumption that the perturbation of the electronic structure caused by the formation of a point defect is confined to the corresponding defect site; i.e., all sites beyond the defect site are considered unperturbed. As a result, the last term in the extensive definition (8) vanishes and the expression for the impurity solution energy in the single-site Green's function technique becomes

$$
E_{\text {sol }}^{\mathrm{ss}-\operatorname{ext}}(0)=E_{B}^{\mathrm{ss}}(0)-E_{B}(1) .
$$

Here, the superscript " ss" refers to quantities determined in the single-site approximation, and obviously $E_{B}^{\mathrm{ss}}(1)=E_{B}(1)$ and $E_{A}^{\mathrm{ss}}(0)=E_{A}(0)$. If, on the other hand, we use the intensive definition (7), we find that the impurity solution energy in the single-site approximation is 


$$
E_{\mathrm{sol}}^{\mathrm{ss}-\mathrm{int}}(0)=E_{B}^{\mathrm{ss}}(0)-E_{B}(1)+\left.\frac{d E_{A}^{\mathrm{ss}}(c)}{d c}\right|_{c=0}
$$

It is now seen that while the renormalization of the electronic structure of the host atoms around the impurity is completely neglected in the single-site extensive definition (10) it is in fact taken into account by the last term in the single-site intensive definition (11). This means that the extensive definition of the impurity solution energy breaks down in the single-site approximation and, as a result, it must be used with caution. In contrast, the intensive definition is still valid and the renormalization term may be obtained in the single-site approximation, for instance, by means of methods based on the CPA, which allow one to calculate the concentration derivatives of the on-site energies. Such calculations have been performed by Johnson et al. ${ }^{19}$ for the impurity solution energy of $\mathrm{Zn}$ in $\mathrm{Cu}$, and further numerical examples presented in Sec. III show that the contribution from the renormalization is substantial even in the case of alloys of neighboring elements in the Periodic Table.

At this point we should mention that it is possible to include in an approximate way the renormalization term, even in the single-site approximation, without calculating the concentration derivatives of the on-site energies. For instance, in the Green's function calculations by Drittler et al. ${ }^{2}$ of impurity solution energies the most important contribution to the renormalization was included in the single-site approximation by means of Lloyd's formula ${ }^{20}$ for the change of the integrated density of states. In the Lloyd formula all changes of the local densities of states are implicitly summed over the infinite crystal volume and as we show in Sec. III D this compensates the errors of the single-site approximation in Green's function calculations to a large degree. However, a similar compensation does not seem possible when Lloyd's formula is used in conjunction with the GPM and similar methods.

\section{Effective chemical potential}

Similar to the impurity solution energy, the effective chemical potential of a completely random alloy at $0 \mathrm{~K}$ may be obtained in two different ways. From the extensive form of the total energy (2) it may be defined as the difference between the total energy of the completely random alloy $A_{1-{ }_{c}} B_{c}$ with either an extra $B$ atom or an extra $A$ at site $i$, i.e.,

$$
\begin{aligned}
\mu(c)^{\mathrm{ext}}= & \left\langle\left.\widetilde{E}_{\mathrm{tot}}\right|_{i=B}\right\rangle-\left\langle\left.\widetilde{E}_{\mathrm{tot}}\right|_{i=A}\right\rangle=\left\langle\epsilon_{i}^{(i=B)}(c)\right\rangle-\left\langle\epsilon_{i}^{(i=A)}(c)\right\rangle \\
& +\sum_{j \neq i}\left\langle\epsilon_{j}^{(i=B)}(c)\right\rangle-\sum_{j \neq i}\left\langle\epsilon_{j}^{(i=A)}(c)\right\rangle=E_{B}(c) \\
& -E_{A}(c)+\sum_{j \neq i}\left[\left\langle\epsilon_{j}^{(i=B)}(c)\right\rangle-\left\langle\epsilon_{j}^{(i=A)}(c)\right\rangle\right],
\end{aligned}
$$

where $\left\langle\boldsymbol{\epsilon}_{j}^{(i=B)}(c)\right\rangle$ and $\left\langle\boldsymbol{\epsilon}_{j}^{(i=A)}(c)\right\rangle$ are the average on-site energies at site $j$ in the completely random alloy with site $i$ occupied by a $B$ or an $A$ atom, respectively.

The intensive definition of the effective chemical potential is

$$
\begin{aligned}
\mu(c)^{\mathrm{int}} \equiv & \frac{d E_{\mathrm{rand}}}{d c}=E_{B}(c)-E_{A}(c)+c \frac{d E_{B}(c)}{d c} \\
& +(1-c) \frac{d E_{A}(c)}{d c}
\end{aligned}
$$

and if we compare with the extensive definition (12), we find

$$
\begin{aligned}
& c \frac{d E_{B}(c)}{d c}+(1-c) \frac{d E_{A}(c)}{d c} \\
& \quad=\sum_{j \neq i}\left[\left\langle\epsilon_{j}^{(i=B)}(c)\right\rangle-\left\langle\epsilon_{j}^{(i=A)}(c)\right\rangle\right],
\end{aligned}
$$

which is a generalization of the relation (9). Again, the physical interpretation is transparent: The weighted change of the on-site energies with concentration is equal to the renormalization of the total energy of the complete system due to the interchange of an $A$ and a $B$ atom in some lattice site. We note that the intensive definition (13) remains unchanged in the single-site approximation, i.e.,

$$
\mu^{\mathrm{ss}-\operatorname{int}}(c)=E_{B}^{\mathrm{ss}}(c)-E_{A}^{\mathrm{ss}}(c)+c \frac{d E_{B}^{\mathrm{ss}}(c)}{d c}+(1-c) \frac{d E_{A}^{\mathrm{ss}}(c)}{d c}
$$

where the last two terms take into account the renormalization of the atoms around the extra $A$ and $B$ atoms positioned at a particular site in the otherwise completely random alloy.

To arrive at the extensive definition in the single-site approximation we note that in this case each site occupied by an alloy component is surrounded solely by the effective medium which may be defined within the virtual crystal approximation (VCA), the average $t$-matrix (ATA) approximation, or the CPA. ${ }^{23}$ This effective medium is homogeneous in the sense that it does not depend on the position of its sites relative to the "defect" site. This means that the last two terms in Eq. (12), which take into account the renormalization of the alloy effective medium due to the presence of an $A$ or a $B$ atom at the specified "defect" site $i$, vanish in the single-site approximation. As a result

$$
\mu^{\mathrm{ss}-\mathrm{ext}}(c)=E_{B}^{\mathrm{ss}}(c)-E_{A}^{\mathrm{ss}}(c),
$$

and as in the case of the impurity solution energy, the extensive definition of the effective chemical potential breaks down in the single-site approximation, because the response of the system to the incorporation of a defect is neglected.

At this point we should mention that the quantity $S_{i}^{(1)}$ defined in the concentration wave formalism ${ }^{21,22}$ is exactly equal to $\mu^{\text {ss -ext }}$ and is also called the "effective chemical potential." However, $S_{i}^{(1)}$ is in fact not a partial molar quantity and is not equivalent to the effective chemical potential defined in the present work. The main difference comes from the fact that while we consider the change of the energy of an alloy due to the interchange of an $A$ and a $B$ atom, $S_{i}^{(1)}$ is the change in the total energy of an alloy due to an infinitessimal change of the concentration of one of the components at some particular site $i$. In this case it is clear that the contribution from the renormalization of the neighboring sites vanishes. $^{22}$ 


\section{Surface segregation energies}

Let us write the Hamiltonian of a system with a surface in the restricted or canonical scheme, which is the basis for calculating the concentration-profile-dependent effective cluster interactions as they are determined in the DCA or GPM:

$$
\begin{aligned}
H\left(\left\{c_{\nu i}\right\}\right)= & E^{(0)}\left(\left\{c_{\nu}\right\}\right)+\frac{1}{N} \sum_{\lambda i} \widetilde{V}_{\lambda}^{(1)}\left(\left\{c_{\nu}\right\}\right) \delta c_{\lambda i} \\
& +\frac{1}{2 N} \sum_{\lambda i} \sum_{\lambda^{\prime} j \neq \lambda i} \widetilde{V}_{\lambda i \lambda j}^{(2)}\left(\left\{c_{\nu}\right\}\right) \delta c_{\lambda i} \delta c_{\lambda^{\prime} j} \\
& +\frac{1}{3 ! N} \sum_{\lambda i} \sum_{\lambda^{\prime} j \neq \lambda i} \sum_{\lambda^{\prime \prime} k \neq \lambda i ; \lambda^{\prime} j} \widetilde{V}_{\lambda i \lambda^{\prime} j \lambda^{\prime \prime} k}^{(3)} \\
& \times\left(\left\{c_{\nu}\right\}\right) \delta c_{\lambda i} \delta c_{\lambda^{\prime} j} \delta c_{\lambda^{\prime \prime} k}+\cdots .
\end{aligned}
$$

Here $N$ is the number of atoms in each layer, $\delta c_{\lambda i}=c_{\lambda i}-c_{\lambda}, c_{\lambda i}$ the occupation numbers in each layer $\lambda$ and site $i, c_{\lambda}=\left\langle c_{\lambda i}\right\rangle \equiv(1 / N) \sum_{i} c_{\lambda i}$ the average concentration of one of the alloy components in layer $\lambda$, and $E^{(0)}\left(\left\{c_{\nu}\right\}\right)$ the energy of the completely random reference system per atom in the layer with fixed concentration profile $\left\{c_{\nu}\right\}$. Finally, $\widetilde{V}_{\lambda}^{(1)}\left(\left\{c_{\nu}\right\}\right), \quad \widetilde{V}_{\lambda i \lambda^{\prime} j}^{(2)}\left(\left\{c_{\nu}\right\}\right), \quad$ and $\widetilde{V}_{\lambda i \lambda^{\prime} j \lambda \prime \prime}^{(3)}\left(\left\{c_{\nu}\right\}\right)$ are the concentration-profile-dependent effective cluster interactions.

As at a fixed alloy concentration profile $\left\{c_{\nu}\right\}$,

$$
\sum_{i} \delta c_{\lambda i}=0
$$

and therefore the contribution from the on-site terms, i.e., $\widetilde{V}_{\lambda}^{(1)}\left(\left\{c_{\nu}\right\}\right)$ in Eq. (17), vanishes. Nonetheless, one may still define an on-site term $\widetilde{V}_{\lambda}^{(1)}$ commonly referred to as a pointenergy interaction, and use Eq. (17) in calculations of concentration profiles. This is possible in the case when $\widetilde{V}_{\lambda}^{(1)}$ and other interactions depend weakly on the concentration profile so that one may vary the concentration profile around the initial values $\left\{c_{\nu}\right\}$ and then redefine the interactions in accordance with the new values $\left\{c_{\nu}^{\prime}\right\}$. In this case, the second term in Eq. (17) does not vanish for those layers $\lambda$ whose final values of $c_{\lambda}^{\prime}$ differ from the initial ones $c_{\lambda}$ since $(1 / N) \sum_{i} \delta c_{\lambda i}^{\prime}=\left(c_{\lambda}^{\prime}-c_{\lambda}\right)$.

The point-energy interaction $\widetilde{V}^{(1)}$ in Eq. (17) is defined as the energy of replacing an $A$ atom by a $B$ atom in a site $i$ of the $\lambda$ th layer in the completely random alloy, i.e.,

$$
\begin{aligned}
\widetilde{V}_{\lambda}^{(1)}\left(\left\{c_{\nu}\right\}\right)= & \left.\frac{\delta \widetilde{E}\left(\left\{c_{\nu}\right\}\right)}{\delta c_{\lambda i}}\right|_{\delta c_{\lambda i}=1} \\
= & \left.\widetilde{E}\left(\left\{c_{\nu i}\right\}\right)\right|_{c_{\lambda i}=1,\left\langle c_{\lambda j}\right\rangle=c_{\lambda},\left\langle c_{\nu i}\right\rangle=c_{\nu}} \\
& -\left.\widetilde{E}\left(\left\{c_{\nu i}\right\}\right)\right|_{c_{\lambda i}=0,\left\langle c_{\lambda j}\right\rangle=c_{\lambda},\left\langle c_{\nu i}\right\rangle=c_{\nu}} \\
= & \frac{\partial E^{(0)}\left(\left\{c_{\nu}\right\}\right)}{\partial c_{\lambda}},
\end{aligned}
$$

where the second line is the extensive definition and the third line the intensive. We note that $\widetilde{V}_{\lambda}^{(1)}$ for $\lambda \in$ bulk is the effective chemical potential of the completely random alloy $A_{1-c} B_{c}$ with $c=c_{\lambda}$, which is

$$
\mu(c)=\widetilde{V}_{\mathrm{bulk}}^{(1)}(c)=\frac{d E^{(0)}(c)}{d c}=\left.\widetilde{V}_{\lambda}^{(1)}(c)\right|_{\lambda \in \text { bulk }} .
$$

The segregation energy of a completely random alloy is defined in terms of the surface energy $E_{\text {surf }}$ as

$$
\begin{aligned}
E_{\lambda}^{\mathrm{segr}} & \equiv \frac{d E_{\text {surf }}}{d c_{\lambda}}=\frac{\partial}{\partial c_{\lambda}}\left[E^{(0)}\left(\left\{c_{\nu}\right\}\right)-\mu \sum_{\lambda}\left(c_{\lambda}-c\right)\right] \\
& =\frac{\partial E^{(0)}\left(\left\{c_{\nu}\right\}\right)}{\partial c_{\lambda}}-\mu .
\end{aligned}
$$

If we now make use of Eq. (20), we find the general relation

$$
E_{\lambda}^{\mathrm{segr}}=\widetilde{V}_{\lambda}^{(1)}\left(\left\{c_{\nu}\right\}\right)-\widetilde{V}_{\mathrm{bulk}}^{(1)},
$$

which links the segregation energy of component $B$ in the $\lambda$ th layer to the point-energy interactions.

In the GPM and DCA one determines a point-energy interaction which corresponds to the extensive definition of the effective chemical potential in the single-site approximation. Hence,

$$
\widetilde{V}^{(1)-\operatorname{GPM}_{(}}(c)=\mu^{\mathrm{ss}-\mathrm{ext}}(c)=E_{B}^{\mathrm{ss}}(c)-E_{A}^{\mathrm{ss}}(c),
$$

where the on-site energies are calculated either within the KKR- or LMTO-CPA methods ${ }^{24}$ in the case of the GPM or in the TB-CPA in the case of the DCA. In these methods the expansion of the total energy in terms of concentration fluctuations on the lattice sites is performed at an alloy composition fixed at the initial value. Often only "band energy" terms are included in Eq. (23) and charge transfer effect neglected.

The segregation energy in the GPM and the DCA becomes

$$
\begin{aligned}
E_{\lambda}^{\mathrm{segr}-\mathrm{GPM}}= & \widetilde{V}_{\lambda}^{(1)-\mathrm{GPM}}\left(\left\{c_{\nu}\right\}\right)-\widetilde{V}_{\mathrm{bulk}}^{(1)-\mathrm{GPM}}(c) \\
= & E_{\lambda}^{B \text {-ss }}\left(\left\{c_{\nu}\right\}\right)-E_{\lambda}^{A-\mathrm{ss}}\left(\left\{c_{\nu}\right\}\right)-E_{\text {bulk }}^{B-\mathrm{ss}}(c) \\
& +E_{\mathrm{bulk}}^{A-\mathrm{ss}}(c),
\end{aligned}
$$

which should be compared with the result of using the intensive definition for $\widetilde{V}^{(1)}$ given in the last line of Eq. (19), i.e.,

$$
\begin{aligned}
E_{\lambda}^{\mathrm{segr}-\mathrm{ss}}= & E_{\lambda}^{\mathrm{segr}-\mathrm{GPM}}+\sum_{\nu^{\prime}}\left(c_{\nu^{\prime}} \frac{\partial E_{\nu^{\prime}}^{B-\mathrm{ss}}\left(\left\{c_{\nu}\right\}\right)}{\partial c_{\lambda}}\right. \\
& \left.+\left(1-c_{\nu^{\prime}}\right) \frac{\partial E_{\nu^{\prime}}^{A-\mathrm{ss}}\left(\left\{c_{\nu}\right\}\right)}{\partial c_{\lambda}}\right) \\
& +\sum_{\nu_{\mathrm{vac}}} \frac{\partial E_{\nu_{\mathrm{vac}}}^{\mathrm{vac}-\mathrm{ss}}\left(\left\{c_{\nu}\right\}\right)}{\partial c_{\lambda}}-\left(c \frac{\partial E^{B-\mathrm{ss}}(c)}{\partial c}\right. \\
& \left.+(1-c) \frac{\partial E^{A-\mathrm{ss}}(c)}{\partial c}\right) .
\end{aligned}
$$


Here the sum over atomic layers is divided into a sum over the surface region, $\nu^{\prime}$, and the vacuum region, $\nu_{\text {vac }}$, and $E_{\nu_{\text {vac }}}^{\text {vacss }}\left(\left\{c_{\nu}\right\}\right)$ is the on-site energy of the vacuum layer $\nu_{\text {vac }}$.

From Eq. (25) it follows that the segregation energy calculated witin the GPA and DCA does not include the effect of the renormalization of the electronic structure on the sites in the neighborhood of the impurity both at the surface and in the bulk. The questions are therefore the following: How large is this effect in real alloys, and can the GPM or the DCA give reliable results for surface segregation energies and segregation profiles?

\section{NUMERICAL ILLUSTRATION: Cu-Ni RANDOM ALLOYS}

To answer the question of the applicability of the GPA and DCA in calculations of segregation profiles we consider a random $\mathrm{Cu}_{c} \mathrm{Ni}_{1-c}$ alloy on an fcc lattice with a fixed lattice parameter of $3.54 \AA$ together with the (100) surface of a $\mathrm{Cu}_{50} \mathrm{Ni}_{50}$ random alloy. The actual calculations were performed by means of the LMTO-CPA method in conjunction with a Green's function technique ${ }^{25}$ in the scalar-relativistic, frozen core, and atomic sphere approximations. ${ }^{1,25}$ Valence electrons were treated self-consistently within the local density approximation (LDA) with the Perdew-Zunger parametrization ${ }^{26}$ of the results of Ceperley and Alder $^{27}$ for the exchange-correlation potential and energy. Integration over the Brillouin zone was performed by the special point technique $^{28}$ with $505 \mathbf{k}$ points in the irreducible wedge (1/ 48th) of the fcc Brillouin zone in the bulk calculations and with $36 \mathbf{k}$ points in the irreducible wedge (1/8th) of the square lattice in the surface calculations. Finally, the screened-impurity model ${ }^{1}$ (SIM) was used to correct the electrostatic one-electron potential and the total energy by including charge transfer effects in the single-site LDA calculations. More details of the calculation and most of the results, e.g., the segregation profile, may be found in Ref. 4.

Although the present type of calculations may treat random alloys with large charge transfer effects ${ }^{1}$ quite accurately, it is important for the accuracy of GPM-type calculations that these effects are small. In fact, the charge neutrality condition for the CPA effective medium $c \Delta q_{A}+(1-c) \Delta q_{B}=0$ results in a large concentration dependence of the on-site energies $E_{A}^{\mathrm{ss}}(c)$ and $E_{B}^{\mathrm{ss}}(c)$ unless the net charges of the alloy components, $\Delta q_{A}$ and $\Delta q_{B}$, are small. Hence, the application of the GPM-type calculations, which often completely neglects charge transfer effects, to systems with large net charges would be highly doubtful on the basis of this alone. However, in the CuNi system the effective charge transfer $\Delta q^{*}=\left|\Delta q_{\mathrm{Cu}}-\Delta q_{\mathrm{Ni}}\right|$ (Ref. 29) is approximately 0.01 electron per atom, assuming equal sphere radii for $\mathrm{Cu}$ and $\mathrm{Ni}$, and the system is expected to be favorable for the GPM-type calculations since we find that the SIM correction to the total energy of the random alloy is only of the order of $0.15 \mathrm{meV}$.

\section{A. Effective chemical potential}

We first consider the correction

$$
\mu(c)^{\mathrm{ss}-\mathrm{cor}}=c \frac{d E_{\mathrm{Cu}}^{\mathrm{ss}}(c)}{d c}+(1-c) \frac{d E_{\mathrm{Ni}}^{\mathrm{ss}}(c)}{d c},
$$
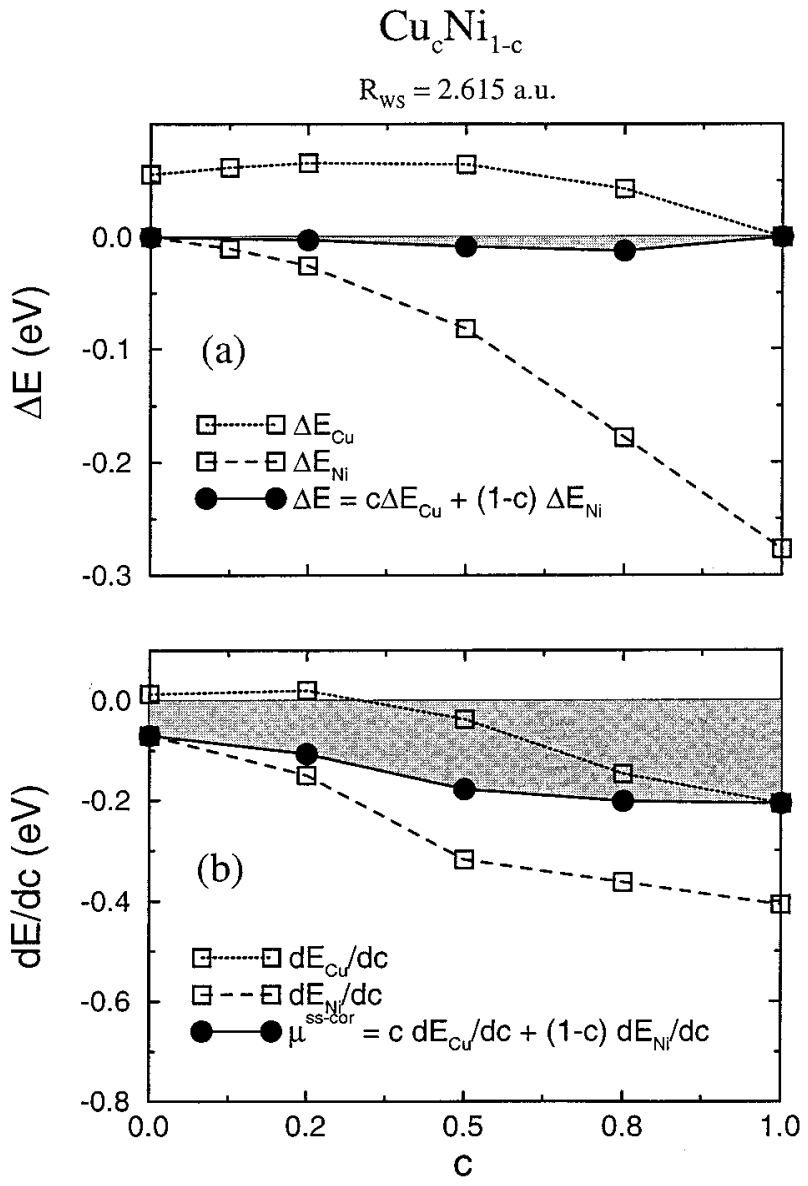

FIG. 1. (a) The deviations of the total energy of random $\mathrm{Cu}_{c} \mathrm{Ni}_{1-c}$ alloys from its average value, $\Delta E(c)$, and the difference between the on-site energies of $\mathrm{Cu}$ and $\mathrm{Ni}$ in the alloys and their values in the pure metals. (b) The correction of the single-site extensive definition $\mu^{\text {ss-cor }}(c)$ and its on-site contributions.

which must be applied to the effective chemical potential in the extensive definition (16) to include the effect of the renomalization of the effective medium; cf. Eq. (15). That is, we compare the GPM-type calculations with the results of Ruban et al. ${ }^{4}$ supplemented by further calculations for the present work. For convenience we consider the difference between the on-site energies $E_{\mathrm{Cu}}^{\mathrm{ss}}(c)$ and $E_{\mathrm{Ni}}^{\mathrm{ss}}(c)$ of $\mathrm{Cu}$ and $\mathrm{Ni}$ in the alloy at some $\mathrm{Cu}$ concentration $c$ and their values in the pure metals, $E_{\mathrm{Cu}}(1)$ and $E_{\mathrm{Ni}}(0)$, i.e.,

$$
\begin{aligned}
& \Delta E_{\mathrm{Cu}}(c)=E_{\mathrm{Cu}}^{\mathrm{ss}}(c)-E_{\mathrm{Cu}}(1) \\
& \Delta E_{\mathrm{Ni}}(c)=E_{\mathrm{Ni}}^{\mathrm{ss}}(c)-E_{\mathrm{Ni}}(0) .
\end{aligned}
$$

Thereby, the deviation of the total energy $E_{\text {alloy }}(c)$ of the random alloy from the average value of the energies of the pure alloy components, $E_{\mathrm{avr}}(c)=c E_{\mathrm{Cu}}(1)+(1-c) E_{\mathrm{Ni}}(0)$, is

$$
\Delta E(c)=E_{\text {alloy }}(c)-E_{\mathrm{avr}}(c)=c \Delta E_{\mathrm{Cu}}^{\mathrm{ss}}(c)+(1-c) \Delta E_{\mathrm{Ni}}^{\mathrm{ss}}(c),
$$

which is the function plotted in Fig. 1(a). In the figure one observes the very interesting feature that while $\Delta E(c)$ is very small over the complete concentration range the on-site 
energies show a strong concentration dependence with $\Delta E_{\mathrm{Ni}}$ reaching almost $-0.3 \mathrm{eV}$ in the $\mathrm{Cu}$-rich alloys. We find that $\Delta E(c)$ is at most $-0.012 \mathrm{eV}$ which is in accordance with the very small calculated mixing energy of the $\mathrm{Cu}-\mathrm{Ni}$ random alloys of the order of $0.014 \mathrm{eV} /$ atom for an equiatomic alloy composition. Note that $\Delta E(c)$ is not equal to the mixing energy since no volume optimization is performed.

As a result of the strong concentration dependence of the on-site energies the correction (26) to the single-site extensive definition of the effective chemical potential in $\mathrm{CuNi}$ alloys is non-negligible as shown in Fig. 1(b). One may argue that the values seen in the figure are very small compared with the values of the actual effective chemical potentials. For instance, the effective chemical potential of the equiatomic random alloy is $-316.42913 \mathrm{eV}$, and even much larger in the soft-core approximation, while the value of the correction for this alloy is only $-0.177 \mathrm{eV}$. However, the correction should be compared with, for instance, the impurity solution energies and the segregation energies, which are of a similar order of magnitude in this system (see below). We conclude that even in systems with a small charge transfer ${ }^{30}$ the GPM-type calculations of partial molar properties will have appreciable errors.

\section{B. Solution energy}

We now consider the solution energy of $\mathrm{Cu}$ in $\mathrm{Ni}$ which we calculate by the CPA method to be $0.15 \mathrm{eV}$ [the experimental value is $0.12 \mathrm{eV}$ (Ref. 31)] and of $\mathrm{Ni}$ in $\mathrm{Cu}$ which we calculate to be $0.03 \mathrm{eV}$ [experimental values are 0.03 and $0.06 \mathrm{eV}$ (Refs. 31,32)]. At the same time we find from Fig. 1 (b) that the correction to the single-site extensive results is approximately $-0.08 \mathrm{eV}$ for $\mathrm{Cu}$ in $\mathrm{Ni}$ and $-0.20 \mathrm{eV}$ for $\mathrm{Ni}$ in $\mathrm{Cu}$, i.e., of the same order of magnitude as the solution energies themselves. Thus, the solution energies of the $\mathrm{CuNi}$ system cannot be calculated in the single-site approximation without the renormalization term. In the single-site Green's function calculations Drittler et al. ${ }^{2}$ found much smaller errors, i.e., -0.02 and $0.03 \mathrm{eV}$, than those inferred from Fig. 1(b). The reason is that these authors used Lloyd's formula and as mentioned earlier thereby implicitly included the renormalization of the band energy term. Had they used the local summation formula instead of Lloyd's formula, the difference would have been much larger and similar to the case of a $\mathrm{V}$ impurity in $\mathrm{Cu}$ discussed in their paper. ${ }^{2}$

\section{Surface segregation}

The segregation energies are determined as the difference between point-energy terms and one might therefore expect some kind of error cancellation in this case. However, such a cancellation does not occur. In Table I we show the surface segregation energies for the first three layers of the (100) surface of $\mathrm{a} \mathrm{Cu}_{50} \mathrm{Ni}_{50}$ random alloy calculated by us directly from Eq. (25) and in the extensive (or GPM) definition (24) compared with the GPM results of Ref. 6 .

In Table I we observe that the present GPM results are in excellent agreement with the GPM results of Drchal et al. ${ }^{6}$ The two sets of GPM results are, however, not consistent with those obtained directly from the intensive definition. In particular, according to the latter, one may expect a strong
TABLE I. The segregation energies for the first three layers of a (100) surface of a $\mathrm{Cu}_{50} \mathrm{Ni}_{50}$ random alloy (in eV).

\begin{tabular}{cccc}
\hline \hline Layer $(\lambda)$ & $E_{\lambda}^{\text {segr-ss }}$ & $E_{\lambda}^{\text {segr-GPM }}($ this work) & $E_{\lambda}^{\text {segr }- \text { GPM }}($ Ref. 6) \\
\hline 1 & -0.360 & -0.3018 & -0.3188 \\
2 & 0.064 & -0.0017 & -0.0027 \\
3 & -0.004 & -0.0056 & -0.0008 \\
\hline \hline
\end{tabular}

segregation of $\mathrm{Cu}$ into the first layer and a weaker $\mathrm{Ni}$ enrichment of the second layer, i.e., an oscillating concentration profile, while according to the GPM segregation energies the concentration profile should be monotonic. Thus, the use of the GPM point-energy interactions leads to a qualitative error in the surface segregation calculations, even in the case of an alloy system with a small charge transfer, which is most favorable for the GPM-type calculations.

\section{Lloyd's formula}

As found by Drittler et al. ${ }^{2}$ the application of Lloyd's formula in the Green's function technique for isolated impurities to a large degree takes account of the renomalization of the electronic structure on the neighboring sites and thereby improves the accuracy of the calculations. It is often assumed that a similar improvement may be found if Lloyd's formula is used in conjunction with the GPM. Unfortunalely, this is not the case as we will now show.

According to definition (see, e.g., Ref. 24) the bandenergy contribution to the point-energy interactions in the GPM is

$$
V_{\mathrm{band}}^{(1)-\mathrm{GPM}}=-\int^{E_{F}}\left[N^{B}(E)-N^{A}(E)\right] d E,
$$

where $N^{B}(E)$ and $N^{A}(E)$ are the local, integrated densities of states for $A$ and $B$ atoms in the alloy. The point energies may be calculated directly from the Green's function or by means of Lloyd's formula. In actual calculations the results of the two approaches should be the same apart from differences in angular momentun convergence. ${ }^{33}$ Hence, if Lloyd's formula does not work there should be no difference between $V_{\text {band }}^{(1)-G P M}$ and $E_{\lambda}^{\text {segr-GPM }}$. This is illustrated by $E_{\lambda}^{\text {segr-GPM }}$ in Table I which in the present work is obtained by direct integration of the Green's function while Drchal et al. ${ }^{6,7}$ use Lloyd's formula. The difference is at most $0.02 \mathrm{eV}$ and may be attributed to differences in numerical details.

\section{CONCLUSION}

We have shown that computational methods which are based on the extensive formulation of the total energy and used in conjunction with the single-site approximation in calculations of partial molar properties of random alloys completely neglect the renormalization of the host effective medium. The alternative single-site intensive formulation does not suffer from this deficiency and yields accurate values for the impurity solution energy, the effective chemical potential, and the segregation energy. We have estimated the magnitude of the terms missing in the extensive formulation and find that even in the CuNi alloy system, where charge transfer effects are small and the interactions between alloy com- 
ponents relatively weak, the missing terms are substantial. In calculations of surface concentration profiles the neglect leads not only to quantitatively but also to qualitatively incorrect results, e.g., monotonic rather than oscillatory profiles. Therefore, one must be careful in applying techniques such as the GPM, the DCA, and the technique developed in Refs. 21 and 22, which are all based on effective interactions determined in the single-site approximation at a fixed alloy composition, i.e., corresponding to the extensive formulation, in calculations of partial molar properties of random alloys.

\section{ACKNOWLEDGMENTS}

The center for Atomic-scale Materials Physics is sponsored by the Danish National Research Foundation.
${ }^{1}$ A. V. Ruban, I. A. Abrikosov, and H. L. Skriver, Phys. Rev. B 51, 12958 (1995).

${ }^{2}$ B. Drittler, M. Weinert, R. Zeller, and P. H. Dederichs, Phys. Rev. B 39, 930 (1989).

${ }^{3}$ Z. W. Lu, S.-H. Wei, A. Zunger, S. Frota-Pessoa, and L. G. Ferreira, Phys. Rev. B 44, 512 (1991).

${ }^{4}$ A. V. Ruban, I. A. Abrikosov, D. Ya. Kats, D. Gorelikov, K. W. Jacobsen, and H. L. Skriver, Phys. Rev. B 49, 11383 (1994).

${ }^{5}$ A. Pasturel, V. Drchal, J. Kudrnovsky, and P. Weinberger, Phys. Rev. B 48, 2704 (1993).

${ }^{6}$ V. Drchal, J. Kudrnovsky, and I. Turek, in Stability of Materials, Vol. 355 of NATO Adanced Study Institute, Series B: Physics, edited by A. Gonis, P. E. A. Turchi, and J. Kudrnovsky (Plenum Press, New York, 1996), p. 355.

${ }^{7}$ V. Drchal, J. Kudrnovsky, A. Pasturel, I. Turek, and P. Weinberger, Phys. Rev. B 54, 8202 (1996).

${ }^{8}$ O. K. Andersen and O. Jepsen, Phys. Rev. Lett. 53, 2571 (1984).

${ }^{9}$ O. K. Andersen, O. Jepsen, and D. Glötzel, in Highlights of Condensed-Matter Theory, edited by F. Bassani, F. Fumi, and M. P. Tosi (North-Holland, New York, 1985).

${ }^{10}$ W. Schweika and A. E. Carlsson, Phys. Rev. B 40, 4990 (1989).

${ }^{11}$ M. Asta, C. Wolverton, D. de Fontaine, and H. Dreyssé, Phys. Rev. B 44, 4907 (1991).

${ }^{12}$ I. A. Abrikosov, A. V. Ruban, H. L. Skriver, and B. Johansson, Phys. Rev. B 50, 2039 (1994).

${ }^{13}$ P. L. Hansen, A. M. Molenbroek, and A. V. Ruban, J. Phys. Chem. (to be published).

${ }^{14}$ A. Christensen, A. V. Ruban, P. Stoltze, K. W. Jacobsen, H. L. Skriver, and J. K. Nbrskov (unpublished).

${ }^{15}$ V. Drchal, J. Kudrnovsky, L. Udvardi, P. Weinberger, and A. Pasturel, Phys. Rev. B 45, 14328 (1992).

${ }^{16}$ M. Brejnak, J. Kudrnovsky, and P. Mordak, Surf. Sci. 307, 821 (1994).

${ }^{17}$ T. Schulthess, R. Monnier, and S. Crampin, Phys. Rev. B 50, 18564 (1994).
${ }^{18}$ H. Dreyssé, L. T. Wille, and D. de Fontaine, Phys. Rev. B 47, 62 (1993).

${ }^{19}$ D. D. Johnson, D. M. Nicholson, F. J. Pinski, B. L. Gyorffy, and G. M. Stocks, Phys. Rev. B 41, 9701 (1990).

${ }^{20}$ P. Lloyd, Proc. Phys. Soc. London 90, 207 (1967); 90, 217 (1967).

${ }^{21}$ B. L. Gyorffy and G. M. Stocks, Phys. Rev. Lett. 50, 4630 (1983).

${ }^{22}$ J. B. Staunton, D. D. Johnson, and F. J. Pinski, Phys. Rev. B 50, 1450 (1994).

${ }^{23}$ J. S. Faulkner, Prog. Mater. Sci. 27, 1 (1982).

${ }^{24}$ A. Gonis, X.-G. Zhang, A. J. Freeman, P. Turchi, G. M. Stocks, and D. M. Nicholson, Phys. Rev. B 36, 4630 (1987).

${ }^{25}$ I. A. Abrikosov and H. L. Skriver, Phys. Rev. B 47, 16532 (1993).

${ }^{26}$ J. Perdew and A. Zunger, Phys. Rev. B 23, 5048 (1981).

${ }^{27}$ D. M. Ceperley and B. J. Alder, Phys. Rev. Lett. 45, 566 (1980).

${ }^{28}$ V. Kumar, D. Kumar, and S. K. Joshi, Phys. Rev. B 19, 1954 (1979).

${ }^{29}$ P. A. Korzhavyi, A. V. Ruban, S. I. Simak, and Yu. Kh. Vekilov, Phys. Rev. B 49, 14229 (1994).

${ }^{30}$ In an attempt to suppress as much as possible the influence of charge transfer effects we have also performed calculations with different atomic Wigner-Seitz radii $S_{\mathrm{Cu}}$ and $S_{\mathrm{Ni}}$ for $\mathrm{Cu}$ and $\mathrm{Ni}$ in the equiatomic alloy. We find that if $S_{\mathrm{Cu}}=1.003 S_{\mathrm{Ni}}$, charge transfer effects disappear. This leads to a reduction of the correction $\mu^{\text {ss-cor }}$ to $-0.068 \mathrm{eV}$ which is still a non-negligible energy.

${ }^{31}$ O. Kubaschevsky and C. B. Alcock, Metallurgical Thermochemistry (Pergamon, Oxford, 1979).

${ }^{32}$ R. Hultgren, P. D. Desai, D. T. Hawkins, M. Gleiser, and K. K. Kelly, Selected Values of Thermodynamic Properties of Metals and Alloys (American Society of Metals, Metals Park, OH, 1973).

${ }^{33}$ X.-G. Zhang and W. H. Butler, Phys. Rev. B 46, 7433 (1992). 